\title{
The effect of cold temperature stress on the viability of rye (Secale cereale L.) microspores
}

\author{
Sylwia Mikołajczyk, Zbigniew Broda, Dorota WeigT \\ Department of Genetics and Plant Breeding, Poznań University of Life Sciences, Poznań, Poland \\ Corresponding author: sylviam@au.poznan.pl
}

\begin{abstract}
Rye has been generally considered to be recalcitrant to the androgenesis induction in vitro. The haploids of Secale cereale are not easily obtainable and the isolated microspore technique application is difficult, thus is performed occasionally, and in a limited number of strains and cultivars. The aim of the conducted experiment was to assess the frequency of mechanical damage in microspores after their isolation in the mortar, and the effect of the pretreatment of spikes at $4{ }^{\circ} \mathrm{C}$ on microspore viability and the course of the culture. The analyses in the isolated microspore culture were conducted on 30 genotypes of rye from two breeding programs and differing in their origin. Spikes with microspores in the unicellular stage were cut and exposed to a temperature of $4{ }^{\circ} \mathrm{C}$ for a period of 2 to 42 days. Microspore observations were conducted immediately after the microspore isolation in order to assess the viability and percentage of the damaged ones. In order to study the mitotic divisions first, cultures of rye microspores were examined after 3 days. The highest microspore viability after isolation was observed for strain $1283 \mathrm{C}-91.9 \%$ and inbred line S1152/10 - 91.8\%, while it was the lowest for $\mathrm{F}_{1}$ hybrid S02779/10 - 79.8\% and strain $1252 \mathrm{E}-84 \%$, respectively. It was found that to maintain high (microspore - to mozna opuscic) viability, microspores must be isolated after low temperature stress $\left(+4^{\circ} \mathrm{C}\right)$ applied for up to 7 days of culture. A considerable extension of the duration of low temperature (36-42 days) resulted in a reduction of the microspore isolation efficiency in rye (a lower number of microspores in the obtained suspension) and a slight reduction of microspore viability.
\end{abstract}

Key words: rye, microspore culture, cold pre-treatment

\section{Introduction}

Rye (Secale cereale ssp. cereale) is a recalcitrant species, in which it is difficult to obtain haploids using in vitro culture. Obtainment and maintenance of in vitro cultures of some plant species is difficult, simply because all the factors required for an effective initiation of androgenesis in these species are not yet known. Factors that are hard to control include endogenous and exogenous factors for the activity of antioxidant enzymes, osmolarity of the culture at the onset of androgenesis, genotypic variations, especially in cross-pollinated plants (rye), response to temperature stress, condition of the donor plant, etc. Due to above-mentioned reasons, it is difficult to identify a method for effective androgenesis induction and regeneration of haploid plants for this species.

\section{Materials and methods}

Analyses of isolated microspore cultures were conducted on 30 rye genotypes (winter forms). Plants of ele- ven $F_{1}$ hybrids and four inbred lines from the Danko Plants Breeding (Danko Hodowla Roślin) and fifteen strains from the Poznan Plants Breeding (Poznańska Hodowla Roślin) were used in the experiment.

After reaching an appropriate development phase, spikes with microspores at the unicellular stage were cut and treated at $4{ }^{\circ} \mathrm{C}$ for a period from 2 to 42 days. The microspore development phase was identified using crushed specimens stained with acetocarmine. Randomly selected inflorescences of the examined plants, containing microspores at the uninucleate stage (Immonen and Anttila, 1998), were surface sterilized using 70\% ethanol and through immersion in $6 \% \mathrm{NaClO}$, were next rinsed in large amounts of sterile distilled water.

From the sterilized spikes, individual spikelets were isolated by removing rachillas. Next, the spikelets were macerated in $0.2 \mathrm{M}$ cold mannitol. Thereafter, they were gently ground in steril mortar, in order to release microspores from anthers. The tissues ground in $0.2 \mathrm{M}$ man- 
nitol solution were filtered through a sterile nylon filter with $80 \mu \mathrm{m}$ mesh size. The mortar was rinsed with $0.2 \mathrm{M}$ mannitol, a sufficient amount to obtain $15 \mathrm{ml}$ filtrate. Sterile test tubes containing the suspension of micropores were centrifuged thrice for $5 \mathrm{~min}$ at $60 \mathrm{~g}$. Test tube contents were suspended in $30 \mathrm{ml}$ of androgenesis induction medium (190-2, Xingzhi and Han, 1984 ) and the obtained microspore suspension was distributed to 10 Petri dishes $(\varnothing 35 \mathrm{~mm})$ at $3 \mathrm{ml}$ each. The dishes were sealed tightly with parafilm and placed in the dark on a shaker at $100 \mathrm{rpm}$. The cultures of microspores were incubated at $27^{\circ} \mathrm{C}$ for 7 days.

Microspore observations were taken directly after isolation in order to assess the viability and percentage of the damaged microspores. Microspore developmental status was evaluated after a 3-day incubation under in vitro conditions, in the 190-2 medium (Xingzhi and Han, 1984). It was done using a reverse microscope in 10 fields of vision and $7 \mathrm{x}$ magnification (eyepiece $10 \mathrm{x}$ objective 0.7 ). The viability of microspores was then examined under an optic microscope in 10 fields of vision at a magnification of $100 \mathrm{x}$.

\section{Results and discussion}

Success in the isolated microspore culture of rye depends on many factors. The most important of these include spike pre-treatment procedure, selection of the microspore developmental stage, donor plant genotype as well as proper culture medium and culture conditions. In order to re-program their development from gametophytic to sporophytic, microspores of plants are exposed to diverse stress factors. Most frequently, inflorescences or anthers are subjected to the action of low or high temperatures. Anthers are often placed in solutions with varying osmotic potential and at a reduced sugar availability, causing sugar starvation, etc. (Shariatpanahi et al., 2006). In order to change the microspore development pathway into sporophytic, spike pre-treatment at $4{ }^{\circ} \mathrm{C}$ is generally applied in microspore cultures of cereals (Jähne and Lörz, 1995).

The technique of isolated microspore culture is applied in breeding programs of Secale cereale L. very occasionally. Thermal stress application $\left(\right.$ at $\left.4{ }^{\circ} \mathrm{C}\right)$ is commonly used to induce androgenesis in anther cultures (Immonen and Anttila, 1999; Tenhola-Roininen et al., 2005 ) and isolated microspore cultures of rye (Guo and
Pulli, 2000; Ma et al., 2004). Thus, for the analyzed 30 genotypes of Secale cereale, low temperature of $4{ }^{\circ} \mathrm{C}$ was selected as the stress factor, with spikes exposed to its action for a period from 0 to 6 weeks.

Microspore culture efficiency in cereals is influenced by the microspore isolation methods from anthers or whole spikes. Based on previously published literature, we may distinguish four techniques to obtain microspores of cereals - cultures of spontaneously released microspores, microspores released by shaking, by maceration, or with the use of a blender (Jähne and Lörz, 1995; Gustafson et al., 1995).

In a study on rye androgenesis (Rakoczy-Trojanowska et al., 1996) two methods of microspore release were employed - a spontaneous and a mechanical release. And, it was observed that the former method yields insufficient number of microspores for an appropriate course of culture, while the latter makes it possible to obtain only a low number of structures comprising several cells. In addition, Some authors successfully isolated microspores of rye, with high microspore survival (up to $96 \%$ ) merely by maceration (Guo and Pulli, 2000; Ma et al., 2004). Deimling and Geiger (1996) indicated that the use of a blender together with maceration, helps to improve the rye microspores culture efficiency.

In our study, the microspores were isolated by maceration of rye anthers in a mortar. The highest microspore viability after isolation was observed for rye strain $1283 \mathrm{C}$ at $91.9 \%$ and inbred line $\mathrm{S} 1152 / 10$ at $91.8 \%$, while it was the lowest for rye $\mathrm{F}_{1}$ hybrid S02779/10, accounting for $79.8 \%$ and strain $1252 \mathrm{E}$ at $84 \%$ (Table 1). The mechanical isolation process of microspores from rye spikelets resulted in their damage, with the percentage of damaged microspores ranging from 8.2 to $20.2 \%$ for microspores from rye genotypes from the Danko Plant Breeding and from 8.1 to $16.2 \%$ for microspores from rye genotypes from the Poznan Plant Breeding. The mean viability of rye microspores after isolation from the analyzed populations from Danko was 87.6\%, while for microspores from rye populations obtained from Poznań was $87.2 \%$.

Hoekstra et al. (1993) characterized different types of barley microspores on the first days of culture. They showed that only microspores with a markedly enlarged diameter divide after a 3-day incubation in a medium that induces androgenesis. In contrast to viable micro- 
Table 1. Mean viability of microspores after isolation from rye spikelet's for analyzed genotypes of Secale cereale L.

\begin{tabular}{l|c|c|c|c|c}
\hline \multicolumn{1}{c|}{ DANKO Plants Breeding } & \multicolumn{3}{c}{ Poznan Plants Breeding } \\
\hline genotype & $\begin{array}{c}\text { viable } \\
\text { microspores } \\
{[\%]}\end{array}$ & $\begin{array}{c}\text { damage } \\
\text { microspores } \\
{[\%]}\end{array}$ & strain & $\begin{array}{c}\text { viable } \\
\text { microspores } \\
{[\%]}\end{array}$ & $\begin{array}{c}\text { damage } \\
\text { microspores } \\
{[\%]}\end{array}$ \\
\hline Inbred line S1038/10 & 85.3 & 14.7 & $1242 \mathrm{~A}$ & 83.5 & 16.5 \\
\hline Inbred line S1152/10 & 91.8 & 8.2 & $1244 \mathrm{~B}$ & 88.2 & 11.8 \\
\hline Inbred line S1156/10 & 90.7 & 9.3 & $1446 \mathrm{E}$ & 88.8 & 11.2 \\
\hline Inbred line S 1174/10 & 83.2 & 16.8 & $1247 \mathrm{C}$ & 88.6 & 11.4 \\
\hline $\mathrm{F}_{1}$ hybrid S02258/10 & 86.8 & 13.2 & $1252 \mathrm{E}$ & 84.0 & 16.0 \\
\hline $\mathrm{F}_{1}$ hybrid S0 2323/10 & 91.4 & 8.6 & $1256 \mathrm{E}$ & 84.9 & 15.1 \\
\hline $\mathrm{F}_{1}$ hybrid S02334/10 & 85.3 & 14.7 & $1259 \mathrm{~A}$ & 86.3 & 13.7 \\
\hline $\mathrm{F}_{1}$ hybrid S02406/10 & 88.5 & 11.5 & $1261 \mathrm{~B}$ & 91.1 & 8.9 \\
\hline $\mathrm{F}_{1}$ hybrid S02507/10 & 90.2 & 9.8 & $1264 \mathrm{D}$ & 86.3 & 13.7 \\
\hline $\mathrm{F}_{1}$ hybrid S02514/10 & 85.1 & 14.9 & $1265 \mathrm{C}$ & 83.8 & 16.2 \\
\hline $\mathrm{F}_{1}$ hybrid S02550/10 & 90.2 & 9.8 & $1273 \mathrm{C}$ & 86.0 & 14.0 \\
\hline $\mathrm{F}_{1}$ hybrid S02742/10 & 85.8 & 14.2 & $1278 \mathrm{E}$ & 85.2 & 14.8 \\
\hline $\mathrm{F}_{1}$ hybrid S02750/10 & 89.7 & 10.3 & $1283 \mathrm{C}$ & 91.9 & 8.1 \\
\hline $\mathrm{F}_{1}$ hybrid S02757/10 & 88.6 & 11.4 & $1287 \mathrm{~A}$ & 88.7 & 11.3 \\
\hline $\mathrm{F}_{1}$ hybrid S02779/10 & 79.8 & 20.2 & $1291 \mathrm{~B}$ & 87.0 & 13.0 \\
\hline Mean & 87.6 & 12.4 & $\mathrm{Mean}$ & 87.2 & 12.8 \\
\hline
\end{tabular}

spores which are round and have granular cytoplasm, non-viable microspores are oval and have shrunk cytoplasm. Similar types of non-viable microspores were observed in this study. In a previous study, it was shown that the first microspore divisions of wheat in in vitro culture may be observed after $48-72 \mathrm{~h}$, while in anthers responding to in vitro conditions $31 \%$ microspores had divisible nuclei (Rybczyński et al., 1991). The abovementioned study prompted us to conduct observations of isolated microspore cultures in rye after a $72 \mathrm{~h}$ incubation on the medium. We did not observe any divisible pores after a 3-day culture. Surviving cells accumulated starch, but did not undertake mitotic activity. Thus shrinking, plasmic cell content images were observed under the microscope.

The recorded results suggest that for an appropriate development of rye microspores employing in vitro cultures, it will be necessary to combine thermal stress, i.e. cold temperature pre-treatment of spikes, with osmotic stress. Some stresses, such as cold treatment and osmotic stress, have been used with success in a variety of lines of rye, whereas many genotypes have a limited ability for androgenesis. In conclusion, we have to admit that the development of a universal method for obtaining rye haploids in a microspore culture is more problematic than in other cereals.

\section{References}

Deimling S., Geiger H.H. (1996) Anther culture in rye: Methodical improvements and genetic analysis. Vortr. Pflanzenzüchtg. 35: 225-235.

Guo Y.-D., Pulli S. (2000) Isolated microspore culture and plant regeneration in rye (Secale cereale L.). Plant Cell Rep. 19: 875-880.

Gustafson V.D., Baenziger P.S., Wright M.S., Stroup W.W., Yen Y. (1995) Isolated wheat microspore culture. Plant Cell Tiss. Org. Cult. 42: 207-213.

Hoekstra S., van Zijderveld M.H., Heidekamp F., van der Mark F. (1993) Microspore culture of Hordeum vulgare L.: the influence of density and osmolality. Plant Cell Rep. 12: 661-665.

Immonen S., Anttila A. (1998) Impact of microspore developmental stage on induction and plant regeneration in rye anther culture. Plant Sci. 139: 213-222. 
Immonen S., Anttila A. (1999) Cold pre-treatment to enhance green plant regeneration from rye anther culture. Plant Cell Tiss. Org. Cult. 57: 121-127.

Jähne A., Lörz H. (1995) Cereal microspore culture. Plant Sci. 109: 1-12.

Ma R., Guo Y.-D., Pulli S. (2004) Comparison of anther and microspore culture in the embryogenesis and regeneration of rye (Secale cereale). Plant Cell Tiss. Org. Cult. 76: 147-157.

Rakoczy-Trojanowska M., Kwaśniak A., Malepszy S. (1996) Factors influencing viability and sporophytic development in vitro of rye (Secale cereale L.) microspores. Vortr. Pflanzenzüchtg. 35: 256-257.
Rybczynski J.J., Simonson R.L., Baenziger P.S. (1991) Evidence for microspore embryogenesis in wheat anther culture. In Vitro Cell Dev. Biol. 27: 168-174.

Shariatpanahi M.E., Bal U., Heberle-Bors E., Touraev A. (2006) Stress applied for the reprogramming of plant microspores towards in vitro embryogenesis. Physiol. Plant. 127: 519-534.

Tenhola-Roininen T., Tanhuanpää P., Immonen S. (2005) The effect of cold and heat treatments on the anther culture response of diverse rye genotypes. Euphytica 145: $1-9$.

Wang X.Z., Hu H. (1984) The effect of potato II medium for triticale anther culture. Plant Sci. Lett. 36:237-239. 